by Goske, the average of the same being practically what he obtained as the highest figure. Goske selects his highest value as the factor for calculation. In using this factor, results would be too low on cocoas to which shells having a lower percentage of heavy constituents were added, and would be too high for those samples to which cocoa shells having a higher percentage were added. The method, it appears to us, would be only approximately accurate where shells having practically that factor had been used. There is no way, however, of determining this point in the examination of any cocoa submitted to the analyst. In determining the accuracy of the method it appears more logical to select the average factor rather than the highest factor, although in the examination of commercial samples it is probably better to take the highest value obtained, because the benefit of the doubt is thereby given to the sample in each case. The table above shows lack of uniformity of results. Samples $12 \mathrm{~A}, \mathrm{I} 2 \mathrm{~B}$ and $\mathrm{I} 2 \mathrm{C}$ contained shells, the factor of which was 52.20, much higher than the average selected for the calculation. In this case it would naturally be expected that the results would be too high. This, it will be seen, is the case. Samples $2 \mathrm{~A}, 2 \mathrm{~B}$ and $2 \mathrm{C}$ contained shells, the factor of which was $2 x .26$, and in this case results would be expected to be too low. This expectation is realized. Samples $7 \mathrm{~A},{ }_{7} \mathrm{~B}$ and ${ }_{7} \mathrm{C}$, however, contained shells, the factor of which was 36.66 , practically the figure used in the calculations. Results are, in the three samples respectively, r.6 per cent. high, 4.9 per cent. low, 2.3 per cent. low. If the highest shell value 52.20 had been used in the formula instead of 37 , it will be seen that in the case of mixture $2 \mathrm{~A}$ the amount of added shells indicated would have been 0 , when in fact 4.5 per cent. of shells had actually been added to the cocoa. It would seem, therefore, that this method does not reliably indicate the addition of smaller quantities than 5 per cent. of cocoa shells and cannot be said to afford any accurate idea of the amount of husks actually placed in the commercial product.

Filsinger and Botticher found that the method of A. Goske gives low results. ${ }^{x}$ U. S. FOOD \& DRUG INSPECTION LABORATORY,
BUfFalo, N. $\mathbf{Y}$.

\section{THE ESTIMATION OF ESSENTIAL OILS.}

\author{
By Charles D. Howard.
}

Received February 10, 1911.

During 1908 the writer published a method ${ }^{2}$ for the determination of essential oils in extracts ind pharmaceutical preparations, involving precipitation and extraction in a Babcock milk bottle, the small quantities of chloroform and ether used being volatilized by rapid evaporation in a water bath. This method was applied to a variety of extracts, including, incidentally, benzaldehyde, although there was no intent to claim that it afforded results of any value in the latter in-

${ }^{1} Z$. offentl. Chem. 16, 311.

2Jour. Am. Chem Soc., 30, 608 (1908). stance. ${ }^{ \pm}$The method was subsequently criticized by Hortvet and West, ${ }^{2}$ the authors claiming that the procedure did not serve to eliminate all of the chloroform and that extraction was not complete.

As a result of further experience, it is admitted that this method sometimes affords erratic results in the case of certain oils and that the average worker is liable to encounter some difficulty in securing concordant and accurate figures. It has proved of value, however, in the case of such oils as lemon, orange, wintergreen and peppermint. A method of this character has the advantages of being simple and quickly carried out, requires no large quantity of solvent, and avoids the difficulties in connection with drying and weighing the oil-the latter, in our experience, proving not inconsiderable.

Based upon the conviction of the writer that a much smaller quantity of solvent than that prescribed by Hortvet and West can be made to serve for complete extraction, the following modification was devised and has been used in this laboratory during the past year with good results, having been applied to most varieties of essential oil preparations. The procedure involves application of the principle, reierred to by the writer in his original paper and since confirmed by Hortvet and West, ${ }^{3}$ that when an ethereal solution of an essential oil is rapidly evaporated, no appreciable loss of oil occurs.

Procedure.-Transfer $20 \mathrm{cc}$. of the extract to a fourounce separatory funnel; in the case of preparations containing more than five per cent of oil, take but ro cc. Add $50 \mathrm{cc}$. of water and (except in the case of oils of the type of cinnamon and clove) two drops of strong hydrochloric acid. Shake out with three portions of ether, using I $5 \mathrm{cc}$, Io cc. and $5 \mathrm{cc}$. After each extraction except the last, the ether solution may be run out into a small flask, which is kept stoppered if a series of determinations is being run simultaneously. The combined ether extracts are washed once with ro cc. of ether-saturated water for removal of the bulk of the alcohol, then cautiously transferred to a ro per cent. milk bottle, rinsing the flask and tip of the funnel with an additional two or three cc. of ether. Attach a bulb tube to the stem of the bottle and connect with a filter-pump, immerse the bottle in nearly boiling water, start the pump and shake with a gentle rotary motion at first. When all danger of spirting has passed, shake violently and toward the last immerse in boiling water for a few seconds, or until the application of a match flame demonstrates the complete elimination of the ether. The removal of most of the latter should require not more than two or three minutes. Finally add cold water and centrifuge. In the case of oils heavier than water, salt solution must be used as the floating agent, except with wintergreen, for which cold sulphuric acid (r:2) may be safely and most conveniently used.

\footnotetext{
State LABoRatory OF HygIENE, CONCORD, N. H.

1 Loc. cit., pp. 608, 610.

2 This Journal, 1, 84 (1909).

3 Loc. cit., p. 88.
} 